Revista Posgrado y Sociedad

Sistema de Estudios de Posgrado

Universidad Estatal a Distancia

ISSN 2215-2172

Costa Rica

revistaposgradoysoci@uned.ac.cr

\title{
La evaluación cualitativa en el campo social y en la educación
}

Qualitative assessment and social field and education

\author{
Ronny W. Gamboa A. \\ Mario E. Castillo S. \\ Universidad Nacional \\ Costa Rica
}

Volumen 13, Número 1

Marzo 2013

pp. $45-60$

Recibido: Setiembre, 2012

Aprobado: Marzo, 2013 


\section{Resumen}

La investigación se ha basado en dos paradigmas principales: el positivista y naturalista. Bajo estos paradigmas se han desarrollo los enfoques cuantitativo y cualitativo, los cuales han generado prejuicios teóricos arraigados sobre las diferencias entre ellos que los han colocado como enfoques antagónicos. La realidad de la investigación social y educativa, en particular, va demostrando cada vez más la insuficiencia de ambos enfoques al ser tomados por separado. En la sociedad actual se ha evidenciado la necesidad de una comprensión cualitativa del ser humano. La evaluación de las prácticas sociales se ha convertido en un medio para lograr dicha comprensión. La evaluación de las realidades sociales y culturales presenta la necesidad de acercarse a lo cotidiano, a las interacciones, a las comunidades y a las negociaciones que se tejen desde los sujetos de esas prácticas sociales. La evaluación cualitativa contribuye a lograr esta comprensión. El interés por los métodos cualitativos en la investigación educativa nace de una insatisfacción con el estilo de las evaluaciones cuantitativas. Por ello, ha existido una tendencia en la evaluación educativa a orientarse por metodologías cualitativas que atiendan al contexto sociocultural de la educación. Respaldar uno u otro enfoque no es una adecuada discusión pues ambos poseen ventajas y desventajas. El presente artículo tiene como propósito presentar una discusión en torno a los aspectos básicos de la evaluación cualitativa en el campo social y en la educación.

Palabras clave: investigación cualitativa, investigación cuantitativa, investigación, evaluación, educación y sociedad. 


\begin{abstract}
Research has been based on two main paradigms: the positivist and naturalist. Under these paradigms have been developing quantitative and qualitative approaches, which have generated theoretical prejudices about the differences between them that have positioned themselves as rival approaches. The reality of social and educational research, in particular, is proving inadequate of both approaches if they are taken separately. In today's society, it has highlighted the need for a qualitative understanding of human beings. The evaluation of social practices has become a means to achieve that understanding. The assessment of social and cultural realities presents the need to approach to everyday situations, to interactions, to communities and to negotiations that are woven from the subjects of these social practices. Qualitative evaluation contributes to this understanding. The interest in qualitative methods in educational research stems from a dissatisfaction with the style of quantitative assessments. Thus, there has been a trend in educational assessment guided by qualitative methodologies that address the sociocultural context of education. Support either approach is not an adequate discussion because both have advantages and disadvantages. The important thing is that any researcher and evaluator know all available methods, both quantitative and qualitative approach, so that, based on the purposes which are held to choose one that will enable the achievement of these goals and results " reliable. " This article aims to present a discussion about basics aspects of qualitative assessment in the social field and in education. At the same time, to show how it contributes to the processes of social and educational research from a qualitative perspective.
\end{abstract}

Keywords: qualitative research, quantitative research, research, evaluation, education and society. 


\section{Introducción}

En los últimos años se ha presentado una gran discusión en torno a la evaluación. Esta situación ha sido descrita por Sandín (2003) como una efervescencia académica, administrativa y política respecto a la evaluación, la cual se ha manifestado por medio de la participación activa de gran cantidad de académicos en trabajos de esta naturaleza, generalmente con colaboración de instituciones político-administrativas, asociaciones, instituciones educativas, entre otros.

Morales (2001) señala que a partir de los diferentes paradigmas éticos, epistemológicos o metodológicos surgidos a lo largo de la historia, es posible identificar dos enfoques evaluativos

- El método cuantitativo, el cual trata de evidenciar el nivel en que se han alcanzado los objetivos propuestos a partir de la aplicación de métodos objetivos. Bajo esta perspectiva los objetivos se expresan por medio de conductas observables y cuantificables.

- El método cualitativo, el cual señala que los resultados finales deben ser complementados con un estudio detallado del desarrollo de los procesos.

Sin embargo, actualmente, tanto en la investigación como en la evaluación, se aboga por una integración de ambos métodos. "La existencia de dos paradigmas de investigación diferentes, no implica una yuxtaposición o exclusión entre ellos. No hay uno mejor que el otro. La clave está en que el investigador sepa tomar partido de acuerdo a sus preguntas y objetivo de investigación" (García, 2010, p. 26)

Ambos modelos han generado una gran discusión en torno a su utilidad y hasta los han catalogado como "rivales". La crítica al primer enfoque surge fundamentada en su incapacidad para abordar algunos aspectos importantes desde el punto de vista social y educativo. En este último, por ejemplo, se hace necesario la identificación de los déficits educativos para superarlos bajo otros modelos de enseñanza y aprendizaje (Morales, 2001). El segundo modelo ha sido criticado por falta de progreso metodológico y de fiabilidad de los métodos para la obtención de los datos y su falta de objetividad, aspecto que se ha tratado de "solucionar" con la triangulación y la inclusión de los diferentes ámbitos relacionados con la evaluación.

Por su parte, Sandín (2003) indica que ante la necesidad de la evaluación de proyectos a gran escala surge la evaluación desde una perspectiva técnica, donde los criterios son, básicamente, el grado de productividad y la eficiencia del programa; el evaluador, externo principalmente, desempeña un rol técnico y posee la función de proporcionar la información sobre la intervención, resultados base para la toma de decisiones, principalmente políticas, relacionadas con futuras aplicaciones o cambios del programa. Sin embargo, la inadecuación de este enfoque, basado en métodos cuantitativos, para la valoración de los programas según los contextos 
sociales en los que se desarrollaban, trajo consigo la implementación de una perspectiva cualitativa en la evaluación.

En el ámbito educativo la evaluación "es la aplicación de las habilidades de investigación para determinar el valor de la práctica educativa" (McMillan \& Schumacher, 2005, p.558). En este sentido los autores señalan que la investigación evaluativa ayuda a tomar decisiones respecto a un tema en particular e incrementa el conocimiento disponible sobre una práctica específica, que permiten planificar y mejorarla o justifican la adopción de otra. Un evaluador se constituye, desde esta perspectiva, como un investigador y un educador cuya labor resulta esencial para el funcionamiento de las instituciones educativas.

Morales (2001) apunta que en educación la investigación cualitativa tiene la función de estudiar los aprendizajes de los alumnos, la labor del profesor, los métodos utilizados, distribución en el aula, recursos didácticos, entre otras, pero partiendo de la realidad compleja de la educación. Ello implica abordar las diferentes variables relacionadas con el contexto en el que aparecen. Desde esta perspectiva se plantea la evaluación "como estrategia de comprensión y valoración de los procesos y resultados de un programa educativo" (Sandín, 2003, p. 89). Sin embargo, algunos afirman que no hay un enfoque mejor que otro y que el uso de uno u otro dependerá del fin perseguido.

El presente artículo tiene como propósito presentar una discusión en torno al debate cuantitativo versus cualitativo y los aspectos básicos de la evaluación cualitativa en el campo social y en la educación. A la vez, pretende mostrar cómo esta contribuye a los procesos de investigación social y educativa desde una perspectiva cualitativa.

\section{La evaluación cualitativa en el campo social}

Delgado (2008) indica que en la sociedad actual se ha evidenciado la necesidad de una comprensión cualitativa del ser humano con base en sus pensamientos y creencias, afectos, actitudes y valores, prácticas, productos simbólicos y significados como constructor de su existencia. La evaluación de las prácticas sociales se ha convertido en un medio para lograr dicha comprensión.

$\mathrm{Al}$ respecto Picado (2002) señala que la evaluación en el ámbito social busca garantizar un mejor cumplimiento de los programas y proyectos, por lo que su objetivo es generar información que pueda ser utilizada en la planificación y ejecución de programas y proyectos de desarrollo social. Por lo tanto, esta evaluación cumple funciones institucionales, sociales, históricas y políticas. 
El autor indica que para realizar una evaluación se requiere de un marco teórico que oriente su planificación. En este sentido, desde la perspectiva cualitativa, esta teoría funciona como un elemento esencial para la reflexión en y desde la práctica evaluada, debido a que la realidad evaluada está constituida por hechos observables, significados, símbolos e interpretaciones elaboradas por los sujetos que intervienen en dichas experiencias evaluadas. Muchas de estas experiencias son evaluadas desde la óptica de una justicia social. En este sentido, House (2005) apunta que los principios de justicia social se utilizan para evaluar si la distribución de los beneficios entre los miembros de una sociedad es apropiada, justa y moral. El contenido de tales evaluaciones, por lo general, consiste de discusiones sobre los conceptos de derechos o necesidades. La justicia social está ligada directamente a la evaluación de los programas sociales y educativos.

Brawerman, Raggio y Ruiz (2007) indican que la formulación de un programa social implica proponer un proceso de cambio cuyos resultados deberán evaluarse.

La idea de cambio está implícita desde el inicio del proyecto o programa que luego será evaluado ya que el hecho de pensarlo está poniendo de manifiesto una necesidad de que algo cambie. Un programa social también puede ser visto como la puesta a prueba de estrategias o metodologías innovadoras de intervención para la transformación. En este mismo sentido, el propio proceso evaluativo implica transformación (Brawerman, Raggio \& Ruiz, 2007, pp. 1-2).

Por lo tanto, apuntan los autores, cuando se comienza a pensar en evaluar un programa en función de la factibilidad de realización de otro, se espera que los resultados de la evaluación brinden elementos para modificar o mejorar la estrategia o metodología de intervención implementada, pues el propio proceso de evaluación debería implicar un aprendizaje institucional.

La evaluación de las realidades sociales y culturales presenta la necesidad de acercarse a lo cotidiano, a las interacciones, a las comunidades $\mathrm{y}$ a las negociaciones que se tejen desde los sujetos de esas prácticas sociales. Así, las realidades socioculturales se comprenden desde su vida cotidiana pues los sujetos, en sus interacciones, redefinen permanente y mutuamente, sus actos (Picado, 2002, p. 51).

Se entiende la evaluación de programas "como un proceso de recogida de información que fomenta la reflexión crítica de los procesos, la transformación de los destinatarios del programa y conduce a la toma de decisiones pertinentes en cada situación específica" (Sandín, 2003, p. 90). Por lo tanto, el análisis se centra en el estudio de las circunstancias personales, sociales, políticas $\mathrm{y}$ económicas relacionadas con la acción propia.

Al respecto Herrera y Olmos (2010) afirman que la evaluación de programas se realiza a través de un proceso de toma de decisiones donde se planifica, examina, se recogen 
datos y se informa sobre el valor o mérito del objeto de evaluación.

La práctica de la evaluación estuvo fuertemente influenciada por el o los paradigmas científicos predominantes. Hasta fines de los sesenta, la formación en materia de investigación de quienes se desempeñaban como evaluadores provocó un claro predominio del enfoque experimental considerado como el único que podía responder a los requerimientos del método científico. Se asoció la evaluación o investigación cuantitativa al paradigma positivista de la ciencia y se la identificó con el enfoque hipotético-deductivo. (Brawerman, Raggio \& Ruiz, 2007, p. 3).

Méndez (2007) apunta que ha habido una evolución en el diseño de evaluaciones que se ha visto reflejado en un cambio en las metodologías y técnicas empleadas, pues de la utilización de técnicas acordes al enfoque deductivo cuyo fundamento está en el empleo de técnicas cuantitativas, basadas en la utilización del método experimental (supone hipótesis de causalidad) se ha pasado a la selección de técnicas propias de enfoques inductivos, principalmente técnicas cualitativas, orientadas a explorar y descubrir la lógica de las relaciones causales partiendo de lo concreto para derivar en comportamientos generales (libre de objetivos puesto que no parte de hipótesis de causalidad).
Debido a ello House (2005) indica que los evaluadores han desarrollado enfoques alternativos, incluidos los estudios cualitativos, meta-análisis, entre otros. Sin embargo, el autor señala que aunque algunos evaluadores no consideraron a los estudios cualitativos como científicos, lo que llevó a intensas discusiones internas acerca de la legitimidad científica de los métodos cualitativos, para el año 2000, la diferencia cuantitativa-cualitativa parecía historia.

El abordaje cualitativo, es particularmente apropiado para dar cuenta de la resignificación de los objetivos de los programas por parte de los funcionarios y técnicos que los implementan, de sus percepciones y creencias. También para comprender las visiones $\mathrm{y}$ opiniones de los destinatarios, que modelan las prácticas respecto de los bienes y servicios que reciben. En todos los casos se entiende que las vivencias y reacciones de los diferentes actores que participan de las intervenciones forman parte de las mismas y contribuyen a sus resultados. (Brawerman, Raggio \& Ruiz, 2007, p. 3).

Filstead (1995) señala que el enfoque cualitativo posee un fundamento humanista que permite entender la realidad social, percibe la vida social como la creatividad compartida de los individuos y descubre el mundo a partir de la coexistencia de múltiples realidades, por lo que los individuos son concebidos como agentes activos 
en la construcción de las realidades en las que se encuentran. Por esta razón, dicho enfoque posee el supuesto sobre la importancia de comprender situaciones desde la perspectiva de los participantes en cada situación, lo que ayudaría a cerrar las brechas en el conocimiento de los acontecimientos sociales como lo señala House (2005). En este sentido, Picado (2002) afirma que toda evaluación posee el interés de encontrar una respuesta a un problema. La autora apunta que al realizar la formulación para la evaluación de un programa, proyecto o servicio social o de desarrollo, esta debe contener los siguientes elementos:

- Identificar el alcance del programa, proyecto o servicio.

- Hacer un panorama de las actividades del programa, proyecto o servicio.

- Describir los intereses y propósitos de la evaluación requerida.

- Conceptualizar el problema y las cuestiones o interrogantes que orientará la evaluación.

- Responder a las siguientes preguntas: ¿El problema podrá ser resuelto en un proceso de evaluación? ¿Puede recogerse la información relevante para abordar el problema planteado? ¿Es significativo el problema? ¿Se dispone de una contestación? ¿Es factible su evaluación? ¿Existe un enunciado concreto del problema? Brawerman, Raggio y Ruiz (2007) indican que la participación de todas las personas que intervienen en el diseño y en la implementación de los programas, así como los destinatarios de este deben ser un elemento central de todo proceso de evaluación social. Por ello, Méndez (2007) apunta que la evaluación debe abarcar todos los elementos relevantes de un programa, lo que requiere la aplicación de una metodología pluralista que combine técnicas cualitativas y cuantitativas. $\mathrm{Al}$ respecto, Picado (2002) señala que los enfoques cualitativos y cuantitativos en la evaluación de proyectos sociales son complementarios pues se requiere de un acercamiento metodológico que permita mostrar las integralidad de los procesos, prácticas $\mathrm{y}$ acciones sociales.

En particular en la evaluación de programas esta combinación permite la consecución de objetivos múltiples atendiendo tanto a los procesos como a los resultados. Enriquece los hallazgos evaluativos profundizando en los motivos de las asociaciones que pueden encontrarse entre variables cuantitativas y sugiriendo hipótesis para explicar la variabilidad entre individuos o grupos. Además permite abordar la cuestión de las creencias, motivaciones o actitudes de la población, difíciles de ser reflejadas cuantitativamente. También facilita la triangulación y acrecienta la comunicabilidad de los resultados. (Brawerman, Raggio \& Ruiz, 2007, p. 4).

Brawerman, Raggio y Ruiz (2007) indican que una evaluación cuantitativa o cualitativa depende de la situación o de los momentos de evolución de un programa e indican que la posibilidad de complementarse o integrarse en un mismo diseño permite aumentar la confiabilidad y credibilidad de los resultados. 


\section{La \\ cualitativa educación \\ evaluación \\ y la}

Soto y Bernardini (2009) señalan que muchos de los principios actuales de la educación se identifican con el naturalismo como, por ejemplo, el proceso de crecimiento psicosomático tomado como base de la educación, la exaltación de la libertad y de los intereses individuales de aprendizaje, la valoración del hombre como individuo concreto dentro de una sociedad determinada, el activismo, la búsqueda de un determinado método de enseñanza válido científica y universalmente.

Pérez (1999) apunta que la evaluación cualitativa aborda la educación desde su realidad. Desde este punto de vista, señala el autor, la enseñanza será un encuentro para la investigación de lo real a partir de la cual se presenten elaboraciones teórico-prácticas que permitan a todos los actores relacionados con la educación construir dicha "realidad". "El interés central de la perspectiva cualitativa en la investigación social y por ende, en la investigación educativa se expresa en: el sujeto, la subjetividad, los significados y los espacios de mediación simbólica" (Delgado, 2008, p. 11).

Filstead (1995) apunta que el interés por los métodos cualitativos en la investigación educativa nace de una insatisfacción con el estilo de las evaluaciones cuantitativas. Por ello, existe una tendencia en la evaluación educativa a orientarse por metodologías cualitativas que atiendan al contexto sociocultural de la educación (Ianni \& Terry, 1995).

La Evaluación Cualitativa no es simplemente la verificación de un conocimiento; más importante aún, es el acercamiento al conocer verdadero que se define en el proceso de enseñar y aprender. El conocer y las posibilidades del enseñar configuran la búsqueda de un aprendizaje significativo basado en el proceso de la investigación como forma de romper con el mundo aparencial. Así, la Evaluación Cualitativa representa investigar la realidad para que sea un encuentro con las bases fundantes del objeto que se estudia. (Pérez, 1999, pp. 10-11).

Pérez (1999) apunta que en educación es necesario un proceso de evaluación cualitativa que respete las elaboraciones del colectivo educativo, y que sea capaz de impulsar una acción transformadora del sujeto en su contexto social-cultural; además debe considerar a la enseñanza desde una visión de saberes no previamente establecidos. Por ello, el autor indica que una educación transformadora debe partir de la crítica de lo real para redireccionar la práctica humana, por lo que la evaluación cualitativa se constituye como un medio para lograr una ruptura con la imposición académica y contribuye con la formación de un ciudadano integral, pues contempla la relación investigación-comunicación para 


\section{Ronny Gamboa \& Mario Castillo}

lograr concientización sobre la realidad.

Por otra parte, House (2005) apunta que la relación de causalidad social es más compleja que lo que la teoría sugiere. Incluso en un mismo programa educativo, hay diferentes profesores en diferentes lugares que producen resultados diferentes. Podríamos tratar de controlar a los profesores, pero hay tantas variables que pueden influir en los resultados que los investigadores no pueden controlar a todos ellos. Por ello la evaluación cualitativa busca comprender el sujeto desde su realidad y sus necesidades.

$\mathrm{Al}$ respecto, Stincone (2009) apunta que la evaluación es uno de los procesos que está estrechamente vinculado a la enseñanza y el aprendizaje, y resulta esencial para valorar los progresos o limitaciones de los educandos, la metodología del docente y el contexto escolar. A pesar de ello, señala la autora, la evaluación frecuentemente se encuentra separada del aprendizaje en sí, reflejándose esta situación aún más en la educación primaria cuyos docentes fueron formados para evaluar de forma cuantitativa en su mayoría.

En el sistema educativo la labor del docente va mucho más allá de la transmisión de conocimientos hacia los educandos; esta debe originarse y basarse en un proceso de investigación que permita la evaluación de ese educando como un individuo integral inmerso en un contexto social.

La evaluación cualitativa de los aprendizajes es asumida por los docentes como un proceso que permite ver no sólo el avance de los niños sino lo que los alumnos pueden expresar tomando en cuenta sus cualidades, es más real, subjetiva y sincera, describe las cualidades de los alumnos a nivel emocional, académico, afectivo en diferentes situaciones, valorando sus potencialidades y respetando sus limitaciones. (Stincone, 2009, p. 12).

La evaluación cualitativa, al ser principalmente descriptiva, permite ir más allá de la valoración académica, lo que le permite al docente evaluador ser más sensible y humano y convertirse en un verdadero investigador de la realidad que rodea a cada educando y cómo influye esa realidad directamente en el proceso de aprendizaje (Stincone, 2009). "Para la investigación social y educativa, el sujeto que interesa es el sujeto en relación al mundo donde vive, mundo producido y construido por él; un mundo lleno de significados que se relaciona con las otras personas. Está mediado por normas, por reglas del lenguaje ordinario." (Delgado, 2008, p. 11).

La evaluación cualitativa puede cumplir una serie de funciones entre las que se destaca: "ser diagnosticadora, si se sitúa en el desarrollo de la estrategia de enseñanza y aprendizaje; adaptadora, cuando facilita información para que se puedan realizar adecuaciones y acomodar los diferentes métodos a los distintos ritmos de aprendizaje; controladora, cuando certifica la cantidad y calidad de las competencias adquiridas" (ChahuánJiménez, 2009, p. 181). 
Delgado (2008) apunta que la investigación cualitativa en educación se presenta como una alternativa que da un giro humanista a la visión de la realidad social y educativa, al ser objeto de múltiples interpretaciones que se caracterizan de acuerdo a un enfoque particular, ya sea reflexivo, crítico o interpretativo, desde una perspectiva microsocial.

Hernández y Moreno (2007) señalan que en educación la evaluación ha estado influenciada por el paradigma cuantitativo donde solamente se tiene en cuenta aspectos descriptivos de la evaluación dejando de lado los explicativos y aspectos de la formación de los estudiantes que no pueden reducirse a un número. Mencionan que bajo el enfoque tradicional, que centra la atención en la enseñanza, la evaluación es reducida a una etapa más del proceso de enseñanza y aprendizaje que enfatiza en los productos que son el resultado de conductas observables y medibles. Por su parte, la evaluación cualitativa centra su atención en el aprendizaje, en las actividades, formas, medios y dinámicas que este produce; tiene en cuenta los aspectos conceptuales, procedimentales y actitudinales de los alumnos; contribuye a propiciar la autonomía del alumno bajo experiencias democráticas y pedagógicas.

Sin embargo, el uso de una u otra dependerá, claro está, de los objetivos que se proponga el docente y de los resultados que pretende obtener. Abogar por una u otra, como ya se mencionó, sería privar a la educación de ciertos resultados que pueden resultar relevantes para la educación y su transformación.

\section{Discusión}

House (2005) apunta que aunque los estudios cuantitativos fueron muy utilizados en distintos campos sociales algunos de ellos no obtuvieron los resultados esperados. Por ello después de años de frustración y de cientos de millones de dólares gastados, los políticos y la mayoría de los evaluadores se desencantaron con estos estudios a gran escala debido a su costo, tiempo y a la falta de resultados definitivos. Debido a ello Brawerman, Raggio y Ruiz (2007) apuntan que en los setenta comenzaron a tener mayor aceptación una variedad de métodos y técnicas cualitativas que habían sido considerados inaceptables y no científicos. Esto debido a que los investigadores cualitativos habían sido criticados porque se había considerado que la recopilación de información realizada por ellos sólo reflejaba las opiniones de un grupo de personas, por lo que estaban "falseados por el juicio humano"; la información cualitativa había sido vista como demasiado subjetiva (House, 2005).

$\mathrm{En} \mathrm{su}$ forma ideal una estrategia cuantitativa sería aquella que apunta a obtener datos numéricos susceptibles de ser sometidos a análisis estadísticos. Una estrategia cualitativa sería aquella que recurre 
fundamentalmente a otro tipo de técnicas de construcción de datos (como la observación en terreno, las entrevistas en profundidad, las historias de vida y en general todo tipo de técnicas que implican una interacción mas o menos prolongada entre el /los sujetos que evalúan y quienes aportan información) dando lugar a la interpretación de significados. Las estrategias mixtas serían aquellas que utilizan combinaciones de ambos grupos de técnicas y métodos en una misma evaluación. (Brawerman, Raggio \& Ruiz, 2007, p. 4).

House (2005) indica que el desarrollo de los estudios cualitativos busca, en algún grado, cambiar la concepción de los valores, a menudo expresada como la dicotomía hecho-valor. Esta dicotomía es la creencia de que los hechos se refieren a una cosa y valores se refieren a algo totalmente diferente. Hechos y valores (las afirmaciones de hecho y las afirmaciones de valor) se mezclan en las conclusiones de los estudios de evaluación.

Ante esta "confrontación" entre lo cualitativo y cuantitativo, Scribano (2000) señala que ha existido un prejuicio teórico arraigado sobre las diferencias entre estrategias cuantitativas y cualitativas que los han colocado como enfoques rivales $\mathrm{e}$ inconmensurables. Hernández, Fernández y Baptista (2006) apuntan que durante varios años se indicó que ambos enfoques eran irreconciliables y opuestos, lo que imposibilitaba mezclarlos. Balestrini (2005) señala que las perspectivas cuantitativa $\mathrm{y}$ cualitativa se han desarrollado separadamente a partir de una red de oposiciones, exclusión y sin canales de comunicación, donde una se ha definido y caracterizado por lo que la otra no es. La autora señala que reconociendo la complejidad de los distintos objetos de estudio y debido a la necesidad de lograr un mayor conocimiento de la realidad humana, ha surgido, principalmente en las Ciencias Sociales, la iniciativa de la integración de los métodos cuantitativos y cualitativos.

Desde esta perspectiva, Morgan (1983), citado en Balestrini (2005), señala una serie de actitudes que pueden plantearse al investigador frente a la dicotomía paradigmática en lo cuantitativo y lo cualitativo: asumir una posición integradora, intentar una síntesis, aceptar una postura de contingencia, de dialéctica, de todo vale.

Cea (1998) señala que en investigación se han distinguido básicamente dos enfoques metodológicos fundamentales: la cuantitativa y la cualitativa; las cuales, según la autora, se diferencian tanto en la estrategia empleada en la recolección de la información como en los procedimientos que se emplean para su análisis. Por ejemplo, en el enfoque cuantitativo la base epistemológica es el positivismo, su énfasis está en la medición objetiva de los hechos, demostración de la causalidad y la generalización de los resultados de investigación, la recolección de datos se da en forma estructurada y sistemática, el análisis de la información se basa en un análisis estadístico y sus resultados se 
centran en la búsqueda cuantitativa de leyes generales de la conducta.

Por su parte, en el enfoque cualitativo se busca la compresión de una realidad social desde los sujetos inmersos en ella. Méndez (2007) apunta que aunque hay una tendencia a elegir datos cuantitativos frente a información de carácter cualitativo, la elección de uno u otro no está relacionado con los datos e informaciones a recabar.

La evaluación se ha convertido, en ambos enfoques, en un medio para la comprensión de la realidad social y educativa, y sus resultados se han orientado a la toma de decisiones respecto a los programas sociales $\mathrm{y}$ educativos. Una evaluación cuantitativa servirá para estimar datos en torno a un programa o modelo educativo mientras que una evaluación cualitativa permitirá determinar si se cumplen los objetivos establecidos en los procesos.

La educación representa un proceso continuo y permanente donde el sujeto adquiere las normas básicas para insertarse dentro de un contexto social determinado, le aporta algunas herramientas para incorporarse en el mundo laboral y tiene como principal objetivo la realización de la persona. Como parte de la comprensión del hecho educativo respecto a la problemática y fundamento en la cual ésta se desarrolla, la investigación educativa y la evaluación cualitativa adquieren una importancia especial como instrumento para la descripción, comprensión, retroalimentación, generación de propuestas y reorientación de este. Abogar por uno $u$ otro método no es una adecuada discusión pues todos poseen ventajas y desventajas. Lo importante es que cualquier investigador $\mathrm{y}$ evaluador conozca todos los métodos disponibles, tanto en el enfoque cuantitativo como cualitativo, para que, basado en los propósitos que se posean, pueda optar por aquel que le permita el logro de estos y la obtención de resultados "confiables".

\section{Referencias}

Balestrini, M. (2005). La integración de los métodos cuantitativos y cualitativos en la investigación social. Ponencia presentada en las I Jornadas de Investigación y Postgrado de la Universidad Nacional Experimental Politécnica de la Fuerza Armada (UNEFA).

Brawerman, J., Raggio, L. \& Ruiz, V. (2007). Abordajes cuanti-cualitativos en la evaluación de programas desde la gestión estatal. Ponencia presentada al IV Congreso Argentino de Administración Pública, Secretaría de Gestión Pública, Asociación de Administradores Gubernamentales (AAEAP). Buenos Aires, Argentina. Recuperado de www.crisolps.org.ar/Metodologia/Ponencia_4_Congreso.doc

Cea, M. (1998). Metodología cuantitativa: Estrategias y técnicas de investigación social. Madrid, España: Editorial Síntesis, S.A.

Chahuán-Jiménez, K. (2009). Evaluación cualitativa y gestión del conocimiento. Educación y Educadores, 12(3), 179-195.

Delgado, F. (2008). Retos actuales de la investigación educativa. Actualidades Investigativas en Educación, 8(3), 1-18. Recuperado de http://revista.inie.ucr.ac.cr/articulos/3-2008/archivos/retos.pdf 


\section{Ronny Gamboa \& Mario Castillo}

Filstead, W. (1995). Métodos cualitativos: Una experiencia necesaria en la investigación evaluativa. En T. Cook y Ch. Reichardt (Eds.), Métodos cualitativos y cuantitativos en investigación evaluativa. Madrid, España: Ediciones Morata, S.L.

García, R. (2010). Utilidad de la integración y convergencia de los métodos cualitativos y cuantitativos en las investigaciones en salud. Revista Cubana Salud Pública, 36(1). Recuperado de http://scielo.sld.cu/scielo.php?script=sci_arttext\&pid=So864-34662010000100004\&lng=es

Hernández, R., Fernández, C. \& Baptista, P. (2006). Metodología de la Investigación (Cuarta Edición). México: McGraw-Hill Interamericana.

Hernández, R. \& Moreno, S. (2007). La evaluación cualitativa: una práctica compleja. Educación y Educadores, 10(002), 215-223.

Herrera, M. \& Olmos, S. (2010). Evaluación de programas educativos. En S. Nieto y M. Rodríguez (Coord.), Investigación y evaluación educativa en la sociedad del conocimiento. Salamanca, España: Ediciones Universidad de Salamanca.

House, E. (2005). Qualitative evaluation and changing social policy. En N. Denzin \& Y. Lincoln (Eds.), The sage handbook of Qualitative Research, $3^{\text {rd }}$ ed. (pp. 1069-1081). California, Estados Unidos: Sage Publications.

Ianni, F. \& Terry, M. (1995). Hacia un acercamiento entre las metodologías cuantitativas y cualitativas. En T. Cook y Ch. Reichardt (Eds.), Métodos cualitativos y cuantitativos en investigación evaluativa. Madrid, España: Ediciones Morata, S.L.

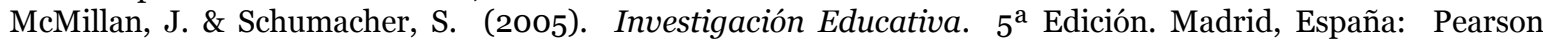
Educación S. A.

Méndez, C. (2007). El cambio de paradigma en evaluación de políticas públicas: el caso de la cooperación al desarrollo. Nuevas políticas públicas. Anuario multidisciplinar para la modernización de las Administraciones Públicas, 3, 145-170.

Morales, J. (2001). La evaluación en el área de educación visual y plástica en la educación secundaria obligatoria (Tesis doctoral). Departamento de Pedagogía Aplicada, Facultad de Educación. Universidad Autónoma de Barcelona.

Pérez, E. (1999). Epistemología de la evaluación cualitativa. Teoría y Didáctica de las Ciencias Sociales, 4, 718.

Picado, M. (2002). ¿Cómo podría delinearse una investigación cualitativa? Revista de Ciencias Sociales, III(97), 67-61.

Sandín, M. (2003). Investigación Cualitativa en Educación: fundamentos y tradiciones. Madrid, España: McGraw-Hill/Interamericana.

Scribano, A. (2000). Reflexiones epistemológicas sobre la investigación cualitativa en Ciencias Sociales. Cinta de Moebio. Revista electrónica de Epistemología en Ciencias Sociales, Núm. 8. Facultad de Ciencias Sociales. Universidad de Chile. Recuperado de http://redalyc.uaemex.mx/pdf/101/10100802.pdf

Soto, A. \& Bernardini, A. (2009). La educación actual en sus fuentes filosóficas. Segunda edición. Decimosexta reimpresión. San José, Costa Rica: EUNED.

Stincone, M. (2009). Evaluación cualitativa de los aprendizajes en la atención a la diversidad. Revista Electrónica de Humanidades, Educación y Comunicación Social (REDHECS), 7. 10-23. Recuperado de http://www.urbe.edu/publicaciones/redhecs/historico/pdf/edicion 7/1-1-evaluacion-cualitativamaria-stincone.pdf 


\section{Nota acerca de los autores}

\section{Ronny Gamboa Araya}

Máster en Matemática Educativa por el Centro de Investigación y Estudios Avanzados del Instituto Politécnico Nacional, México. Licenciado en la Enseñanza de la Matemática por la Universidad Nacional. Se ha desempeñado como director, investigador y académico de la Escuela de Matemática de la Universidad Nacional.

\section{Correo electrónico rgamboa@una.cr}

\section{Mario Castillo Sánchez}

Doctor en Educación por la Universidad Estatal a Distancia. Máster en Estadística por la Universidad de Costa Rica. Egresado de la Licenciatura en la Enseñanza de la Matemática por la Universidad Nacional. Docente de la Escuela de Matemática de la Universidad Nacional y de la Escuela de Salud Pública de la Universidad de Costa Rica.

Correo electrónico mario.castillo.sanchez@una.cr 